\title{
Un nouvel acte de Guillaume le Bâtard, duc de Normandie (18 juin 1066)
}

Résumé

Depuis la parution en 1961 du Recueil des actes des ducs de Normandie de 911 à $\underline{1066}$, c'est un devoir pour les historiens de signaler et de publier tout acte qui a pu échapper aux recherches du savant éditeur, Marie Fauroux. Or, malgré l'ambitieux travail de recensement et d'édition engagé depuis une bonne trentaine d'années autour des actes de la Normandie ducale, seule une poignée de copies ignorées d'actes déjà connus par ailleurs a jusqu'à présent été retrouvée. La découverte récente d'un nouvel acte ducal pour la période jusqu'en 1066 mérite donc une présentation approfondie, d'autant plus qu'il s'agit d'un acte du plus célèbre des ducs normands, donné en faveur de la cathédrale d'Avranches, l'une des institutions ecclésiastiques les plus négligées de l'historiographie normande. Le présent article suivra donc un double objectif : celui de mettre à disposition des chercheurs le texte de ce nouvel acte, le premier à être découvert depuis plus de cinquante ans, et celui d'améliorer notre connaissance de l'activité ducale dans une région dont l'histoire pendant la première moitié du $\mathrm{XI}^{\mathrm{e}}$ siècle reste encore assez obscure.

Richard Allen

Membre associé de la faculté d'histoire (Université d'Oxford)

St Peter's College

Oxford OX1 2DL

Angleterre

richard.allen@spc.ox.ac.uk 
Le Recueil des actes des ducs de Normandie de 911 à 1066, publié par Marie Fauroux il y a plus de cinquante années, constitue toujours un instrument de travail indispensable à tous ceux qui s'intéressent à l'histoire de la Normandie ducale ${ }^{1}$. Développé à partir d'un catalogue des actes qui avait été le sujet d'une thèse soutenue à l'École des chartes en $1950^{2}$, le recueil contient les textes de 234 actes délivrés, confirmés ou souscrits par les premiers ducs normands, à partir de la « fondation » du duché en 911, jusqu'à la veille de la conquête de l'Angleterre. Le rassemblement et l'édition de ces textes, qui faisaient jusque-là l'objet de recherches à la fois sporadiques et incomplètes, ont dès lors permis à des chercheurs de s'interroger sur plusieurs aspects de l'histoire des premiers temps de la Normandie. L'importance de l'ouvrage, honoré en 1962 d'une médaille de l'Académie des Inscriptions et BellesLettres $^{3}$, n'échappait pas aux spécialistes qui en ont rendu compte pendant les années qui ont suivi sa publication. Le travail leur a paru remarquable par son érudition, par le soin avec lequel il a été mené à bien et, avant tout, par l'exhaustivité des recherches déployées. D'après Michel Nortier, par exemple, « les longues recherches de l'auteur ... donnent la garantie qu'aucun acte ni aucune copie importante pour l'établissement du texte n'a pu lui échapper $»^{4}$, tandis que Jacques Boussard fait remarquer «que la recherche des copies est si complète qu'il est tout à fait improbable qu'on puisse y apporter des compléments $»^{5}$.

Témoignage supplémentaire de la qualité du travail effectué, ces constats, faits il y a plus d'un demi-siècle, se sont avérés largement corrects. En effet, malgré l'ambitieux travail de recensement et d'édition engagé depuis une bonne trentaine d'années par

\footnotetext{
${ }^{1}$ Marie Fauroux, Recueil des actes des ducs de Normandie de 911 à 1066, Caen, 1961 (Mémoires de la Société des antiquaires de Normandie, 36).

${ }^{2}$ Marie Le Roy-Ladurie, Catalogue des actes des ducs de Normandie de 911 à 1066, thèse de l'École des chartes, 1950, dactyl. ; résumé dans École nationale des chartes, positions des thèses... 1951, p. 111-112.

${ }^{3}$ L'ouvrage fut couronné par la $1{ }^{\text {ère }}$ médaille du concours des Antiquités de la France : «Rapport sur le concours des Antiquités de la France pour $1962 »$ dans Comptes rendus des séances de l'Académie des Inscriptions et Belles-Lettres, t. 106, 1962, p. 219-222.

${ }^{4}$ Michel Nortier, « Compte rendu de l'ouvrage : M. Fauroux, Recueil des actes des ducs de Normandie

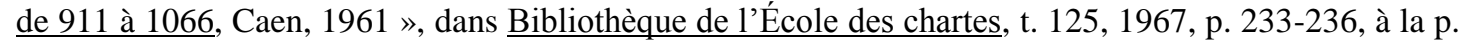
234.

5 Jacques Boussard, «Compte rendu de l'ouvrage: M. Fauroux, Recueil des actes des ducs de Normandie de 911 à 1066, Caen, 1961 », dans Journal des savants, 1965, no 4, p. 661-664, à la p. 662. Des sentiments semblables sont exprimés dans les comptes rendus de Joseph R. Strayer (dans

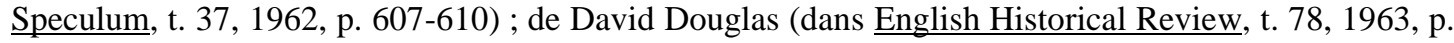
731-732) ; de Walther Kienast (dans Historische Zeitschrift, t. 199, 1964, p. 468-473) ; et de Raymonde Foreville (dans Annales de Normandie, t. 15, 1965, p. 594-596).
} 
plusieurs spécialistes autour des actes de la Normandie ducale ${ }^{6}$, et le dépouillement systématique de nombreux fonds d'archives qui en a résulté, seule une poignée de copies ignorées par Marie Fauroux ont jusqu'à présent été retrouvées ${ }^{7}$. La découverte récente d'un nouvel acte ducal pour la période jusque 1066, inconnu jusqu'ici, mérite donc une présentation approfondie, d'autant plus qu'il s'agit d'un acte de Guillaume le Bâtard (1035-1087), le plus célèbre des ducs normands, donné en faveur de la cathédrale d'Avranches, l'une des institutions ecclésiastiques les plus négligées de l'historiographie normande. Le présent article suivra donc un double objectif : celui de mettre à disposition des chercheurs le texte de ce nouvel acte, le premier à être découvert depuis plus de cinquante ans, et celui d'améliorer notre connaissance de l'activité ducale dans une région dont l'histoire pendant la première moitié $\mathrm{du} \mathrm{XI}^{\mathrm{e}}$ siècle reste encore assez obscure ${ }^{8}$.

C'est en préparant une édition des actes des évêques d'Avranches, actuellement en cours d'achèvement pour la série du Corpus des actes épiscopaux normands $\left(\mathrm{XI}^{\mathrm{e}}\right.$ -

\footnotetext{
${ }^{6}$ Signalons, à titre d'exemple, la base de données SCRIPTA (Site caennais de recherche informatique et de publication des textes anciens), dont une première version, riche de plus de 8500 actes normands antérieurs au XIVe siècle a été mise en ligne en février 2017.

${ }^{7}$ Michaël Bloche, dans son édition du chartrier de l'abbaye de Fécamp, signale l'existence de trois copies (deux actes du duc Richard II et un acte du duc Robert) et d'une charte originale (acte du duc Richard II) ignorées par Marie Fauroux (Michaël Bloche, Le chartrier de l'abbaye de la Trinité de Fécamp : étude et édition critique, 928/929-1190, thèse de l'École des chartes, 2012, dactyl., t. II, $\mathrm{n}^{\text {os }} 6$, $11,12,20)$. Pierre Bauduin, en revanche, a retrouvé une transcription et une (mauvaise) photocopie de photo aux Archives de la Seine-Maritime d'un acte du duc Richard II pour l'abbaye de Saint-Wandrille que signalait Yves Poncelet. Notons également que le texte partiel de sept actes ducaux (des ducs Richard I ${ }^{\text {er }}$, Richard II et Guillaume le Bâtard) figure parmi les fragments du Cartulaire d'Aganon de l'abbaye de Saint-Père de Chartres. Ces fragments, encore dans un état trop fragile lors de la publication du Recueil, viennent d'être restaurés et numérisés dans le cadre du projet À la recherche des manuscrits de Chartres et sont actuellement consultables sur la Bibliothèque virtuelle des manuscrits médiévaux (BVMM) : Ibid., $\mathrm{n}^{\text {os }} 2$ [Bibl. mun. Chartres, ms. 1061 (H.I.50), t. 1, fragments 12A-B (BVMM nos 23-24), t. 2, fragment 24A (BVMM no 115)] ; 29 [ms. 1060 (H.I.49), fragment 26A $\left(\mathrm{BVMM} \mathrm{n}^{\circ}\right.$ 51), ms. 1061 (H.I.50), t. 1, fragment 7A (BVMM no 13)] ; 32 [ms. 1060 (H.I.49), fragment 15B (BVMM $\mathrm{n}^{\circ}$ 30), ms. 1061 (H.I.50), t. 1, fragments 13A-B (BVMM $\left.{ }^{\text {os }} 25-26\right)$ ] ; 50 [ms. 1060 (H.I.49), fragments 26A-B (BVMM no 51-52), ms. 1061 (H.I.50), t. 2, fragment 6B (BVMM no 80)] ; 117 [ms. 1061 (H.I.50), t. 1, fragment 11B (BVMM n ${ }^{\circ}$ 22), ms. 1061 (H.I.50), t. 1, fragment 8A (BVMM, n $\left.{ }^{\circ} 15\right)$ ] ; 146 [ms. 1061 (H.I.50), t. 2, fragments 16A-B (BVMM nos 99-100)] ; 147 [ms. 1061 (H.I.50), t. 2, fragments 9A-B (BVMM nos $85-86)]$. Un acte inclus par erreur dans le Recueil a aussi été dénoncé : Kathleen Thompson, «Une confirmation supposée de Guillaume le Bâtard », dans Annales de Normandie, t. 34, 1984, p. 411-412.

${ }^{8}$ Les années récentes ont heureusement vu un renouvellement des études consacrées à l'histoire de la ville d'Avranches et de l'Avranchin pendant l'époque ducale : Richard Allen, «Un évêque et sa ville : les évêques d'Avranches de 990 à 1134 », dans Revue de l'Avranchin et du pays de Granville, t. 86, 2009, p. 1-49; id., «Robert Cénalis et l'histoire épiscopale d'Avranches de 1100 à 1253 », dans Annales de Normandie, t. 61, 2011, p. 9-24 ; Claude Groud-Cordray, «Hasculf de Saint-James et le prieuré de Sacey : les enjeux d'une fondation et l'ascension d'un seigneur frontalier dans l'Avranchin (fin XI'-début XII ${ }^{\mathrm{e}}$ siècle) », dans Annales de Normandie, t. 63, 2013, p. 27-51.
} 
$\underline{\text { XIII }}^{\mathrm{e}}$ siècle $)^{9}$, que le nouvel acte ducal vient d'être découvert. Masse documentaire de premier ordre pour l'histoire du futur département de la Manche, les actes épiscopaux avranchais sont restés très largement inexploités par les médiévistes depuis les travaux des érudits des $\mathrm{XVIII}^{\mathrm{e}}$ et $\mathrm{XIX}^{\mathrm{e}}$ siècles. Cette méconnaissance tient largement à une histoire archivistique marquée par des catastrophes. Face à un diocèse qui avait été abandonné pendant plus d'un siècle à la suite des incursions scandinaves du $\mathrm{IX}^{\mathrm{e}}$ siècle, les évêques du $\mathrm{XI}^{\mathrm{e}}$ et du XII ${ }^{\mathrm{e}}$ siècle s'efforcèrent de reconstituer la richesse de leur cathédrale, mais elle fut à nouveau dévastée par les Bretons au XIII ${ }^{\mathrm{e}}$ siècle, par les Anglais au XV', et par les protestants en $1562^{10}$. Une autre catastrophe, d'une nature très différente, s'est abattue, il y a deux siècles, sur la cathédrale d'Avranches : déjà partiellement ruinée avant la Révolution française, elle disparut complètement au début du XIX ${ }^{\mathrm{e}}$ siècle, et on ne la connaît aujourd'hui que par des dessins ${ }^{11}$, des plans, et par les résultats des fouilles menées dans les années $1970^{12}$. La perte de ce monument historique est compensée par la survie de quelques manuscrits appartenant à la bibliothèque médiévale de la cathédrale, dont l'un est un cartulaire ${ }^{13}$. Mais les destructions causées par la Révolution, et celles qui furent infligées aux archives de la Manche par les bombardiers alliés en $1944^{14}$, représentent un véritable traumatisme pour le patrimoine écrit médiéval de la région, et en particulier pour ce qui concerne l'ancien diocèse d'Avranches ${ }^{15}$.

Le résultat de tout cela est que les copies effectuées à l'époque moderne par des spécialistes ou par des érudits locaux, mêmes celles dont la transcription est souvent imparfaite, ont aujourd'hui une valeur historique bien plus importante qu'au temps de

\footnotetext{
${ }^{9}$ Corpus des actes épiscopaux normands $\left(\mathrm{XI}^{\mathrm{e}}-\mathrm{XIII}{ }^{\mathrm{e}}\right.$ siècle), dir. Grégory Combalbert, Véronique Gazeau et al., édition papier et numérique, Caen, à paraître. Sur l'édition des actes des évêques d'Avranches, voir Richard Allen, "Les actes des évêques d'Avranches, ca. 990-1253 : esquisse d'un premier bilan », dans Tabularia «Études », t. 12, 2012, p. 63-106.

${ }^{10}$ R. Allen, « Un évêque et sa ville... », p. 1-49; id., « Robert Cénalis... », p. 14.

${ }^{11}$ Maylis Baylé, «Les évêques et l'architecture normande au XI ${ }^{\mathrm{e}}$ siècle », dans Les évêques normands du XI ${ }^{\mathrm{e}}$ siècle (Actes du colloque de Cerisy-la-Salle, 30 septembre-3 octobre 1993), éd. Pierre Bouet et François Neveux, Caen, 1995, p. 151-172, à la p. 159.

12 Daniel Levalet, «La cathédrale Saint-André et les origines chrétiennes d'Avranches », dans Archéologie médiévale, t. 12, 1982, p. 107-153.

${ }^{13}$ Bibl. mun. Avranches, ms. 206 (cartulaire connu sous le nom de Livre vert).

14 Yves Nédélec, «Répertoire des bibliothèques et archives de la Manche », dans Revue du département de la Manche, t. 4, 1962, p. 357-444, aux p. 394-395.

${ }^{15}$ Grâce aux efforts de Natalis de Wailly, ancien directeur de l'École des chartes, plus de 1500 chartes des abbayes de Savigny et de Mortain ont été transférées en 1839 de la sous-préfecture de Mortain aux Archives nationales (Béatrice Poulle, « Les sources de l'histoire de l'abbaye cistercienne de Savigny au diocèse d'Avranches », dans Revue Mabillon, n.s., t. 7 (= t. 68), 1996, p. 105-125). Parmi ces pièces emportées à Paris figurent une soixantaine de chartes des évêques d'Avranches de 1134 jusqu'en 1253.
} 
leur production. On a déjà montré par ailleurs comment la Hierarchia Neustriæ, écrit par l'évêque d'Avranches Robert Cénalis (1532-1560) dans le deuxième tiers du XVI siècle, contient de précieux renseignements, non seulement pour l'histoire avranchaise, mais également pour celle du duché normand ${ }^{16}$. À l'autre extrémité du spectre, les copies réunies au XIX et au début du XX ${ }^{\mathrm{e}}$ siècle pour l'édition des $\underline{\text { Chartes }}$ et diplômes, qui sont conservées aujourd'hui à l'Institut de France ${ }^{17}$, renferment la transcription de plusieurs chartes avranchaises détruites en 1944, dont certaines sont méconnues, voire totalement inconnues ${ }^{18}$. Il en va de même pour les copies effectuées par Johannes Ramackers, conservées aujourd'hui à l'Institut Historique Allemand à Paris ${ }^{19}$. Signalons enfin les fonds d'érudits qui se trouvent actuellement aux Archives départementales de la Manche, tel le fonds Victor Gastebois, entré aux archives par voie d'achat en 2012 et constitué d'une quarantaine de cartons contenant des notes de recherches et des documents originaux concernant la région de Mortain $^{20}$, ainsi que la sous-série $6 \mathrm{~J}$ au même dépôt, qui regroupe des notes et archives d'érudits, dont celles de François-Nicolas Dubosc (1839-1879) et de François Dolbet (1879-1911), anciens archivistes de la Manche.

La Bibliothèque municipale d'Avranches ne manque pas elle non plus de copies d'érudits importantes. Parmi celles-ci signalons particulièrement celles qu'a laissées le chanoine Charles Guérin (1624-1711) ${ }^{21}$; ces copies, faites par lui-même ou par d'autres, ont été acquises en 1883 par l'historien du diocèse d'Avranches, l'abbé Émile-Auber Pigeon (1829-1902), et reliées (probablement par les soins de Pigeon) en un volume factice, doté d'une pagination récente, qui figure aujourd'hui à la

\footnotetext{
${ }^{16}$ R. Allen, « Robert Cénalis... », p. 9-24.

${ }^{17}$ Paris, Académie des Inscriptions et Belles-Lettres, carton 40.

${ }^{18}$ Voir, par exemple, l'acte d'Achard, évêque d'Avranches (1161-1170), jadis conservé en original à Saint-Lô dans le fonds de l'abbaye du Mont Saint-Michel, traitant d'un accord survenu entre celle-ci et l'abbaye de Marmoutier. Aucun inventaire de ce fonds n'avait malheureusement été publié avant la 1944, et il n'en existe aucune autre copie ni, semble-t-il, aucune mention. Une édition critique se trouve dans R. Allen, «Les actes d'Avranches... », Annexe, n 1, p. 99.

${ }^{19}$ Paris, Deutsches Historisches Institut, Nachlaß Ramackers, Deleg. Richter Touraine, Anjou, Maine, et Deleg. Richter Normandie.

${ }^{20}$ Arch. dép. Manche, 134 J (non classé).

${ }^{21}$ Sur Charles Guérin, voir la courte notice biographique qui figure dans les annales d'Avranches dites de 1648 (Émile-Auber Pigeon, éd., «Chronique d'Avranches aux XVII ${ }^{\mathrm{e}}$ et XVIII ${ }^{\mathrm{e}}$ siècles », dans Mémoires de la Société d'archéologie, littérature, sciences et arts des arrondissements d'Avranches et de Mortain, t. 7, 1885, p. 220-224). Ce Guérin ne doit pas être confondu avec un autre Charles Guérin, qui vivait au XIX siècle et qui a aussi laissé des copies de documents (voir Dominique Perrin, «Chalandrey dans les registres paroissiaux du Mesnil-Thibault», dans Revue de l'Avranchin et du pays de Granville, t. 72, 1995, p. 201-242 et 281-306), ni avec le docteur Louis-Julien Guérin, mort en 1843, qui a arraché le crâne de saint Aubert des mains des révolutionnaires.
} 
Bibliothèque municipale dans le fonds de documents achetés par la ville aux héritiers de l'abbé Pigeon ${ }^{22}$. Connu depuis la fin du XIX ${ }^{\mathrm{e}}$ siècle sous le titre de $\underline{\text { Acta sanctæ }}$ $\underline{\text { Ecclesiæ Abrincensis }}^{23}$, ce manuscrit, que Marie Fauroux croyait perdu au XVIII ${ }^{\mathrm{e}}$ siècle ${ }^{24}$, a été récemment remis au jour par Nicholas Vincent dans le cadre de sa nouvelle édition des actes d'Henri II d'Angleterre (1154-1189) ${ }^{25}$, avec pour résultat que l'attention des spécialistes, longtemps accaparée par les seuls documents issus d'archives diocésaines, s'est portée à nouveau vers la relecture et l'édition de plusieurs textes méconnus ou inconnus ${ }^{26}$.

Parmi les nombreux documents transcrits ou recueillis par le chanoine Guérin figure une pancarte en forme de charte récapitulative, par laquelle Jean d'Ivry, évêque d'Avranches (1060-1067), et futur archevêque de Rouen (1067-1079), énumère les possessions de sa cathédrale. D'abord mis en lumière vers la fin du XIX ${ }^{\mathrm{e}}$ siècle par l'abbé Pigeon ${ }^{27}$, cet acte est d'une importance capitale pour notre compréhension de l'histoire du diocèse au début $\mathrm{du} \mathrm{XI}^{\mathrm{e}}$ siècle. Il nous renseigne non seulement sur la nature et l'importance des biens de la cathédrale, mais offre également un bon échantillonnage des donateurs qui ont contribué à la formation du temporel à la charnière de l'an mil. Les interventions ducales y sont assez nombreuses et c'est pour cette raison que la pancarte est citée à trois reprises par Marie Fauroux parmi sa liste des « diplômes perdus ou supposés ${ }^{28}$. L'édition de Pigeon fut faite d'après une copie prise par le chanoine Guérin, qui a lui-même travaillé à partir d'une copie d'un vidimus, jadis conservé aux archives diocésaines mais aujourd'hui perdu, donné sous le sceau de l'officialité d'Avranches, le 10 juillet $1377^{29}$. La récente redécouverte du manuscrit Guérin a naturellement suscité un renouveau d'intérêt pour

\footnotetext{
${ }^{22}$ Bibl. mun. Avranches, fonds Pigeon, ms. 45 (26bis).

$23 \mathrm{La}$ couverture du manuscrit porte la mention: "Guérin, manuscrit unique. Le diocèse d'Avranches ». Le titre de Acta sanctæ Ecclesiæ Abrincensis, celui qu'utilisa Pigeon pour citer le manuscrit, se trouve en tête de la première page des notes de Guérin.

${ }^{24}$ M. Fauroux, Recueil des actes..., p. 398.

${ }^{25}$ The letters and charters of King Henry II (1154-1189), éd. Nicholas Vincent et al., Oxford, à paraître, $\mathrm{n}^{\text {os }} 88,1856,1857,1864,1885,2438$. Je remercie le professeur Vincent de m'avoir communiqué son édition avant sa publication.

${ }^{26}$ Voir, par exemple, Jörg Peltzer, «Portchester, les évêques d'Avranches et les Hommet (11001230) », dans Annales de Normandie, t. 56, 2006, p. 463-482; R. Allen, « Un évêque et sa ville... », p. 1-49 ; id., « Robert Cénalis... », p. 9-24.

${ }^{27}$ Le texte de la pancarte figure dans sa « reconstitution » du cartulaire perdu d'Avranches, le Livre blanc: Émile-Auber Pigeon, Le diocèse d'Avranches, sa topographie, ses origines, ses évêques, sa cathédrale, ses églises, ses comtes et ses châteaux, Coutances, 1888, t. II, p. 666-668.

${ }^{28}$ M. Fauroux, Recueil des actes..., p. 24, n. 24, p. 25-26, n. 29, p. 27, n. 33 et 37.

${ }^{29}$ Bibl. mun. Avranches, fonds Pigeon, ms. 45 (26bis), p. 451-453.
} 
la pancarte, et une édition critique, faite sur la même copie de Guérin, en a été publiée en $2009^{30}$.

Mais bien qu'importante, la copie de Guérin est loin d'être impeccable : outre un certain nombre de fautes de transcription, elle ne reproduit pas intégralement le texte du vidimus, s'arrêtant de manière abrupte après les trois premiers mots des clauses finales («In cujus rei »). Heureusement et contrairement à ce que l'on croyait auparavant, ce n'est pas la seule copie de la pancarte qui se trouve dans le manuscrit Guérin : une autre transcription, dont l'existence a jusqu'ici échappé à l'attention tant des érudits que des spécialistes, est reliée juste avant la copie de Guérin. Il s'agit d'une copie du XVI $\mathrm{I}^{\mathrm{e}}$ siècle, faite d'après une copie authentique du 10 avril 1540, aujourd'hui perdue, collationnée sur le vidimus du 10 juillet 1377, par les tabellions royaux Pierre Gaudin et Lancelot Philippes (une traduction contemporaine est aussi reliée avec elle $)^{31}$. Du point de vue paléographique, le texte est d'une écriture cursive rapide, pleine d'abréviations et de ligatures, souvent très difficile à déchiffrer, ce qui explique sans doute pourquoi il serait jusqu'ici passé inaperçu de tous ceux qui se sont occupés du manuscrit Guérin. Mais, mises à part ces difficultés, la copie nous offre une version plus complète du vidimus, tout particulièrement en ce qui concerne les clauses finales, dont la transcription mérite, en raison de son importance, d'être publiée en entier :

«In cujus rei testimonium presenti vidimus fecimus apponi sigillum curie Abrincen(sis), prius facta collatione cum originali. Datum anno Domini millesimo $\mathrm{CCC}^{\mathrm{o}}$ septuagesimo septimo, die ultima jullii, et au bas estoit escript : Signum Guill(elm)i (une croix) comitis. Signum (une croix) Malthildis comitisse. Signum (une croix) Roberti comitis. Signum (une croix) Richardi fratris. Signum (une croix) Maurilii archiepiscopi. Signum (une croix) Balduini. Signum (une croix) Joannis Abrincen(sis) episcopi. Signum (une croix) Rogerii Montis Grivou. Icelle lettre a simple queue et cire verd. Fait par copp(ie) soulz les seingz manuelz de nous Pierres Gaudin et Lancelot Philippes, tabellions pour le roy, nostre syre, aud(it) lieu jouxte l'original auquel avons collationné ceste presente coppie de mot après mot, signe et consigne en seing, sceau et escripture. Et pour plus grande approbation y a esté mis et apposé les sceaux aux

\footnotetext{
${ }^{30}$ R. Allen, « Un évêque et sa ville... », $\mathrm{n}^{\mathrm{o}}$ II, p. 38-44.

${ }^{31}$ Bibl. mun. Avranches, fonds Pigeon, ms. 45 (26bis), p. 448-450. Les feuillets de la traduction sont intercalés entre p. 449 et 450 et ne sont pas numérotées.
} 
oblig(ations) de lad(ite) vicon(té), le dixme jour d'avril, après Pasques, l'an mil cinq centz quarante. Signez Gaudin, Lancelot, chacun nom et paraphe et scellé en cire verd. »

Ce qui frappe tout d'abord est bien évidemment la liste de témoins. Alors que l'acte était jusque-là tenu pour un acte épiscopal, la souscription du duc Guillaume le Bâtard implique que la pancarte doit désormais être considérée, selon les trois catégories d'actes établies par Marie Fauroux (chartes ducales au sens vrai du terme ; chartes de particuliers confirmées par le duc, souvent par simple souscription ; procès-verbaux de jugements rendus par le duc ou d'accords passés devant la cour ducale) $)^{32}$, comme un acte ducal. Parmi les autres souscripteurs, on voit figurer la comtesse Mathilde († 1083), ainsi que Robert Courteheuse (c. 1050-1134), fils aîné du duc, Richard († 1069/1074), son frère, l'archevêque Maurille de Rouen (1055-1067) et l'évêque Jean d'Avranches.

Avant d'en venir à l'analyse des conséquences de ce changement de perspective, commençons par rappeler très brièvement ce que l'on sait actuellement des circonstances entourant la rédaction de la pancarte. Comme nous l'avons déjà noté, le diocèse d'Avranches avait beaucoup souffert des invasions scandinaves qui bouleversèrent la province ecclésiastique de Rouen au $\mathrm{IX}^{\mathrm{e}}$ siècle : la cathédrale avait été abandonnée, les églises rasées, les reliques dispersées. Après une vacance du siège de plus d'un siècle, la succession épiscopale avranchaise se rétablit enfin vers la fin du $X^{\mathrm{e}}$ siècle avec l'arrivée de l'évêque Norgod (c. 990-1017/c. 1022), dont on sait peu de chose $^{33}$. L'épiscopat de son successeur, Maugis (c. 1017/c. 1022-c. 1026), n'a duré que quelques années, bien qu'il semble avoir réalisé la construction de grandes parties de l'église cathédrale ${ }^{34}$. Son successeur, Hugues (c. 10281055/1060), était un membre important de l'entourage ducal, et cette position privilégiée lui a permis de reconstituer le patrimoine de sa cathédrale, comme en témoigne la pancarte : c'est à la demande de l'évêque («prece et hortatu Hugonis venerabilis antistitis ») que le duc Robert le Magnifique (1027-1035) a donné à la cathédrale l'église Saint-Gervais dans la banlieue d'Avranches, ainsi que les églises des Gens, de Céaux, de Vessey, de la Croix-Avranchin, de Villiers, de Beuvron et

\footnotetext{
${ }^{32}$ M. Fauroux, Recueil des actes..., p. 36-37.

${ }^{33}$ R. Allen, «Un évêque et sa ville... », p. 5-16.

${ }^{34}$ M. Baylé, « Les évêques et l'architecture... », p. 158.
} 
de Vains ${ }^{35}$, ainsi que divers domaines avec leurs dîmes, tonlieux et moulins. De même, entre 1035 et 1060, le duc Guillaume a donné à la cathédrale les églises de Saint-Senier-sous-Avranches, d'Appilly, d'Orceil, de Saint-Pierre-Langers, de Chantore et de Frigabulgam, qui reste non identifiée, tandis que, à la demande («precatu ») de l'évêque, il donnait également à la cathédrale toute la terre tenue dans la région par Garnier, frère de Thierry, hostiarius. Plus intéressant, le duc Robert donna à la cathédrale « la dîme du tonlieu du pagus d'Avranches » («decimam totius telonei Abrincensis pagi »), ce qui non seulement montre que les ducs de Normandie avaient conservé l'administration financière carolingienne, mais prouve également le rôle administratif important d'Hugues dans la région ${ }^{36}$. Toutes ces donations ont considérablement accru la puissance économique de l'évêque dans son diocèse, alors que la donation de Vains sera employée au XII ${ }^{\mathrm{e}}$ siècle par l'un des successeurs d'Hugues pour former le principal bénéfice ecclésiastique sur lequel sera fondé le doyenné d'Avranches ${ }^{37}$.

Or, c'est surtout avec l'élection de l'évêque Jean d'Ivry que l'on peut constater un effort concerté pour rétablir tous les aspects de l'autorité épiscopale. Jean était le fils de Raoul, comte d'Ivry, frère utérin du duc Richard ${ }^{\text {er }}$ (943-996); il appartenait donc à l'une des familles normandes les plus puissantes, dont les membres avaient une expérience considérable dans les régions frontalières du duché ${ }^{38}$. Il n'est donc pas étonnant que le duc ait choisi un tel homme pour occuper ce diocèse de confins : sa présence à Avranches marquait les limites occidentales du duché, et donnait une cohérence à la principauté normande. Homme énergique, l'évêque Jean s'est engagé à donner également une cohérence au temporel de sa cathédrale. Malgré en effet les efforts de ses prédécesseurs, le désordre archivistique occasionné par les raids scandinaves a duré longtemps, comme en témoigne la pancarte elle-même. D’après l'exposé de l'acte, l'évêque Jean, « ayant pris connaissance des chartes de [son] église

\footnotetext{
${ }^{35}$ Pour les identifications administratives de ces communes et de ceux qui suivent, voir l'édition de la pancarte dans l'annexe ci-après.

${ }^{36}$ Lucien Musset, «Recherches sur le tonlieu en Normandie à l'époque ducale », dans Autour du pouvoir ducal normand, $\mathrm{X}^{\mathrm{e}}-\mathrm{XII}{ }^{\mathrm{e}}$ siècles, Caen, 1985 (Cahier des Annales de Normandie, 17), p. 61-76, à la p. 67.

37 Richard Allen, «Five charters concerning the early history of the chapter at Avranches », dans Tabularia «Documents », t. 8, 2008, p. 1-33; id., «Five charters concerning the early history of the chapter at Avranches : an edition revisited », dans Tabularia «Documents », t. 10, 2010, p. 37-44.

${ }^{38}$ Sur sa carrière, voir id., " "A proud and headstrong man”: John of Ivry, bishop of Avranches and archbishop of Rouen, 1060-79», dans Historical Research, t. 83, 2010, p. 189-227.
} 
et y ayant trouvé de grandes confusions dans les possessions de celle-ci ... [a] fait au mieux les corrections qui s'imposaient, en collationnant très soigneusement les chartes qui, mélangées avec d'autres, étaient par trop fautives ${ }^{39}$. Tout comme la grande charte confirmative rédigée quelques années plus tôt pour la cathédrale voisine de Coutances, qui eut aussi à souffrir des Vikings ${ }^{40}$, la pancarte avranchaise est en premier lieu un acte rendu nécessaire par la perte et la confusion des archives antérieures du diocèse. Mais sa forme fait d'elle beaucoup plus qu'une simple liste de donations. Comme beaucoup d'autres pancartes et chartes confirmatives normandes $\mathrm{du} \mathrm{XI}^{\mathrm{e}}$ siècle, elle possède un caractère presque narratif. En dehors de l'exposé circonstancié du contexte, le texte est ponctué d'allusions historiques : la mention de la visite du duc Robert le Magnifique à Jérusalem; le transfert à Saint-James de la foire autrefois tenue à la Croix-Avranchin; l'achèvement d'un parc à gibier par l'évêque Jean «à grands frais » («cum pecunia ») ${ }^{41}$. La pancarte s'inscrit donc dans une tradition diplomatique normande par laquelle les institutions religieuses du duché se sont efforcées pendant ces années non seulement de remettre en ordre leur patrimoine et d'en obtenir la confirmation ducale, mais aussi de créer un document historique sur un aspect de la vie de leur communauté ${ }^{42}$. Rappelons également que l'évêque Jean était un prélat réformateur important et influent. Son épiscopat se caractérise par un effort concerté pour rétablir tous les aspects de l'autorité épiscopale dans le diocèse, et c'est sans doute aussi le même objectif que vise, en partie, la rédaction de la pancarte ${ }^{43}$.

Mais si le besoin pratique et la réforme ecclésiastique constituent le contexte au sens large de la rédaction, la question qui se pose à la lumière de la liste de témoins est

\footnotetext{
39 «... ego Joannes quamvis indignus sancte Abrincensis ecclesie episcopus, cartas ecclesie nostre respiciens magnamque terrarum confusionem in eis inveniens ... que pluribus cartulis confuse titubabant nimium colligens summa veritate correximus ».

${ }^{40}$ La situation géographique du Cotentin l'exposa à des ravages fréquents, et le dernier évêque résident fut assassiné à Saint-Lô en 890. Peu de temps après l'église rouennaise devint le nouveau siège de la cathédrale de Coutances, et grâce à une donation du "duc » Rollon, les évêques s'installèrent dans l'ancienne église de Saint-Sauveur de Rouen, bientôt rebaptisée en l'honneur de saint Lô. Sur le retour de l'institution épiscopale au $\mathrm{XI}^{\mathrm{e}}$ siècle, voir Julie Fontanel, « La réorganisation religieuse sous Guillaume le Conquérant : le cas de l'église de Coutances », dans Revue de l'Avranchin et du pays de Granville, t. 77, 2000, p. 189-208.

${ }^{41}$ Sur le parc construit par Jean, voir Marie Casset, Les évêques aux champs : châteaux et manoirs des évêques normands au Moyen Âge, Caen, 2007, p. 335-344.

${ }_{42}$ À ce sujet, voir David Bates, «Les chartes de confirmation et les pancartes normandes du règne de Guillaume le Conquérant », dans Pancartes monastiques des XI $\mathrm{e}^{\mathrm{e}} \mathrm{XII}{ }^{\mathrm{e}}$ siècles, éd. Michel Parisse, Pierre Pégeot et Benoît-Michel Tock, Turnhout, 1998, p. 95-109, à la p. 102.

${ }^{43}$ R. Allen, «"A proud and headstrong man”... », p. 189-227.
} 
celle de savoir si l'on peut maintenant placer la pancarte, dont les dates extrêmes sont celles de l'épiscopat de Jean d'Ivry, dans un contexte historique beaucoup plus précis. Toute tentative d'élaborer une réponse à cette question se heurte au problème de l'identification de deux des témoins : Baudouin et Roger de Monte Grivou. Pour ce qui est de Roger, la première hypothèse qui se présente est qu'il provenait du petit hameau de Montgriveul ${ }^{44}$, dans la Mayenne, qui est attesté ailleurs sous la forme Mons $\underline{\text { Grivou }}^{45}$. Situé près de la frontière entre la Normandie et le Maine, ce hameau se trouve à l'ombre des châteaux d'Ambrières et de Gorron ${ }^{46}$, dont la construction se situe pendant les guerres normanno-angevines des années 1050-1060. L'organisation militaire de ces fortifications, qui permettent au duc Guillaume d'asseoir son autorité tout au long de cette zone stratégique, nous échappe largement à cette époque, mais nous savons qu'elles furent placées sous l'autorité du comte de Mortain ${ }^{47}$, frère utérin du duc et seigneur le plus considérable du diocèse d'Avranches. Peut-on en conclure que Roger fut rattaché à l'un de ces châteaux et qu'il faisait donc partie de l'entourage comtal qui s'est rendu à une réunion de la cour ducale tenue dans le sillage de la conquête du Maine en 1063 ? On ne saurait déclarer que cela soit impossible, d'autant plus que l'un des hommes mortinais rattaché au château de Gorron à cette époque a été identifié ailleurs ${ }^{48}$; toutefois cela semble peu probable. Non seulement Roger de Montgriveul est inconnu par ailleurs, mais nous ne connaissons pas d'autres mentions de ce toponyme avant le début du XIII ${ }^{\mathrm{e}}$ siècle ${ }^{49}$. C'est pourquoi nous croyons pouvoir identifier Roger de Monte Grivou avec Roger II de Montgommery († 1094), vicomte d'Hiémois, dont le toponyme (Montis Gomerii) semble avoir été mal transcrit par le

\footnotetext{
${ }^{44}$ Le Haut et Le Bas Montgriveul, Mayenne, cant. Gorron, comm. Oisseau.

${ }^{45}$ Nécrologe-obituaire de la cathédrale du Mans, éd. Gustave Busson et Ambrose Ledru, Le Mans, 1906 (Archives historiques du Maine, 7), p. 161.

${ }^{46}$ Montgriveul est situé environ $12 \mathrm{~km}$ au sud-ouest d'Ambrières-les-Vallées (Mayenne, cant. Gorron) et $14 \mathrm{~km}$ au sud-est de Gorron (Mayenne, chef-lieu de cant).

${ }^{47}$ Sur les châteaux de Gorron et d'Ambrières, voir Gérard Louise, La seigneurie de Bellême, $\mathrm{X}^{\mathrm{e}-\mathrm{XII}}$ siècles : évolution des pouvoirs territoriaux et construction d'une seigneurie de frontière aux confins de la Normandie et du Maine à la charnière de l'an mil, Flers, 1990-1991 (Le Pays Bas-Normand, 199202), t. II, p. 191, 205. Sur l'apparition des châteaux dans cette région au cours de cette période, voir Daniel Pichot, Le Bas-Maine du Xe au XIII ${ }^{\mathrm{e}}$ siècle : étude d'une société, Laval, 1995 (La Mayenne, archéologie, histoire. Supplément, 7), p. 133-147. Sur l'organisation militaire du comté de Mortain, voir Jean Pouëssel, «Les structures militaires du comté de Mortain ( $\mathrm{XI}^{\mathrm{e}}$ et XII ${ }^{\mathrm{e}}$ siècles) », dans Revue de l’Avranchin et du pays de Granville, t. 58, 1981, p. 11-74, 81-156.

${ }^{48}$ Il s'agit d'un certain Geoffroi, fils de Rivallon : Katharine Keats-Rohan, « Le rôle des bretons dans la politique de la colonisation normande de l'Angleterre, c. 1042-1135», dans Mémoires de la Société d'histoire et d'archéologie de Bretagne, t. 73, 1996, p. 181-215, aux p. 201-202.

${ }^{49} \mathrm{La}$ mention de la dîme de Montgriveul (Monte Grivou) qui se trouve dans le nécrologe-obituaire de la cathédrale du Mans, cité ci-dessus, date de la charnière de l'an 1200. Pour d'autres mentions, voir Lucien Beszard, "Notes de toponymie mayennaise (suite)», dans Bulletin de la Commission historique et archéologique de la Mayenne, t. 33, 1917, p. 52-68, à la p. 59.
} 
copiste. Non seulement son rang élevé correspond-il mieux à ceux des autres souscripteurs, mais Roger tient une place considérable dans les souscriptions des actes ducaux $^{50}$, et il se trouve à plusieurs reprises parmi les témoins aux côtés du duc, de sa famille et des membres de l'épiscopat normand (fig. 1).

L'identification du témoin nommé Baudouin, en revanche, semble n'offrir, à première vue, qu'une impasse. Face à l'absence de tout titre ou d'autre qualificatif, on serait tout d'abord tenté de conclure qu'il n'était qu'un homme de rang mineur. Le recours à d'autres sources montre cependant que l'attribution de titres par les scribes était assez irrégulière et que les grands n'en portaient pas toujours dans les actes. Les personnes nommées Baudouin figurent, en outre, assez rarement parmi les témoins des actes ducaux à cette époque : on n'y retrouve que Baudouin de Gacé, Baudouin Filleul, et deux autres Baudouin, non identifiés, qui apparaissent tous dans des actes de la période avant 1060-1066 ${ }^{51}$. Constatons également que Baudouin de Meulles, fils de Gilbert, comte de Brionne, compte parmi les donateurs énumérés dans la pancarte d'Avranches. Or, la position de la souscription, coincée entre celles de l'archevêque de Rouen et de l'évêque d'Avranches, nous amène à croire qu'il faut voir en ce témoin une autre figure de haut rang liée à la cour ducale. Il s'agit de Baudouin, évêque d'Évreux (1066-1070), ancien chapelain du duc ${ }^{52}$. L'absence de son titre épiscopal s'explique par le fait que le copiste a d'abord écrit «Signum (une croix) Balduini Abrincen(sis) episcopi », avant d'ajouter «Signum (une croix) Joannis » en interligne, tout en négligeant apparemment de faire précéder ces mots de «Ebroicensis episcopi ». Ce ne serait certainement pas la première fois que la similitude entre les mots latins pour Avranches et Évreux, et surtout entre leurs formes abrégées («Abr'» et «Ebr'»), aurait servi pour introduire de confusion dans une copie d'érudit ${ }^{53}$.

L’intervention de l'évêque Baudouin est un élément d'importance capitale pour nous éclairer sur le contexte historique dans lequel la pancarte avranchaise a été rédigée.

\footnotetext{
${ }^{50}$ M. Fauroux, Recueil des actes..., p. 62.

51 Ibid., $\mathrm{n}^{\text {os }} 124,126,134,137,146$.

52 Baudouin comptait parmi les hommes de confiance du duc Guillaume : Lucien Musset, «Une voie privilégiée d'accès à l'épiscopat dans le monde anglo-normand: la chapelle du duc-roi, v. 1050-v. 1150 », dans L'évêque dans l'histoire de l'Église. Actes de la $7^{\mathrm{e}}$ rencontre d'Histoire religieuse tenue à Fontevraud les 14 et 15 octobre 1983, Angers, 1984, p. 51-62.

${ }^{53}$ Voir, par exemple, Paris, Bibl. Sainte-Geneviève, ms. 1651, p. 172-173.
} 


\begin{tabular}{|c|c|c|}
\hline Fauroux & Date & signa semblables à ceux de la pancarte d'Avranches \\
\hline 99 & 1042,20 avril & Guillaume, duc ; Mathilde. \\
\hline 107 & $\begin{array}{l}1046 / 1047 \text { ou } \\
1048 ?\end{array}$ & $\begin{array}{l}\text { Guillaume, duc ; Mauger, archevêque de Rouen ; Guillaume, évêque } \\
\text { d'Évreux ; Yves, évêque de Sées. }\end{array}$ \\
\hline 120 & v. 1050 & Guillaume, duc ; Mathilde ; Guillaume, évêque. \\
\hline 122 & 1050 & Guillaume, duc ; Mauger, archevêque de Rouen ; Hugues, évêque. \\
\hline 133 & 1054, 25 décembre & $\begin{array}{l}\text { Guillaume, duc ; Mauger, archevêque de Rouen ; Hugues, évêque } \\
\text { d'Avranches. }\end{array}$ \\
\hline 137 & $\begin{array}{l}\text { 1055, septembre ou } \\
\text { octobre }\end{array}$ & $\begin{array}{l}\text { Guillaume, duc ; Maurille, archevêque de Rouen ; Gervais, évêque } \\
\text { du Mans ; Guillaume, évêque. }\end{array}$ \\
\hline 140 & $1049 / 1058$ & Guillaume, duc ; Hugues, évêque. \\
\hline 141 & 1052 env./1058 & $\begin{array}{l}\text { Guillaume, duc ; Mathilde ; Guillaume, fils du duc ; Odon, évêque } \\
\text { de Bayeux ; Geoffroi, évêque de Coutances. }\end{array}$ \\
\hline 142 & 1059 & Guillaume, duc ; Mathilde. \\
\hline 145 & $1046 / 1060$ & Guillaume, duc ; Hugues, évêque ; Guillaume, évêque. \\
\hline 163 & 1050 env./1064 & Geoffroi, évêque de Coutances. \\
\hline 194 & 1050 env./1066 & Guillaume, duc ; Mathilde. \\
\hline 195 & 1050 env./1066 & Guillaume, duc ; Mathilde ; Odon, évêque de Bayeux. \\
\hline 198 & 1050 env./1066 & Mathilde. \\
\hline 199 & $1051 / 1066$ & Guillaume, duc ; Mathilde ; un fils du duc. \\
\hline 208 & $1055 / 1066$ & $\begin{array}{l}\text { Guillaume, duc ; Mathilde ; Maurille, archevêque de Rouen ; } \\
\text { Guillaume, évêque d'Évreux. }\end{array}$ \\
\hline 218 & $1059 / 1066$ & Guillaume, duc ; Mathilde ; Robert Courteheuse, fils du duc. \\
\hline 219 & 1060 env./1066 & $\begin{array}{l}\text { Guillaume, duc ; Mathilde ; Robert Courteheuse, fils du duc ; } \\
\text { Guillaume, évêque d'Évreux. }\end{array}$ \\
\hline 220 & $1060 / 1066$ & $\begin{array}{l}\text { Guillaume, duc ; Jean, évêque d'Avranches ; Guillaume, évêque } \\
\text { d'Évreux. }\end{array}$ \\
\hline 226 & $1065 / 1066$ & Guillaume, duc ; Mathilde ; Robert Courteheuse, fils du duc. \\
\hline 228 & 1066 & Jean, évêque d'Avranches. \\
\hline 229 & 1066 & $\begin{array}{l}\text { Guillaume, duc; Mathilde ; Robert Courteheuse, fils du duc ; } \\
\text { Maurille, archevêque de Rouen; Jean, évêque d'Avranches; } \\
\text { Baudouin, évêque d'Évreux. }\end{array}$ \\
\hline 230 & 1066 & $\begin{array}{l}\text { Guillaume, duc ; Mathilde ; Robert Courteheuse, fils du duc ; } \\
\text { Hugues, évêque de Lisieux. }\end{array}$ \\
\hline 231 & 1066,18 juin & $\begin{array}{l}\text { Guillaume, duc; Mathilde; Robert Courteheuse, fils du duc; } \\
\text { Richard, fils du duc; Maurille, archevêque de Rouen; Hugues, } \\
\text { évêque de Lisieux; Odon, évêque de Bayeux ; Jean, évêque } \\
\text { d'Avranches ; Baudouin, évêque d'Évreux. }\end{array}$ \\
\hline 232 & $\begin{array}{l}1066 \text { (entre } 1 \text { e } 27 \\
\text { mai et le } 16 \text { juillet) }\end{array}$ & $\begin{array}{l}\text { Guillaume, duc ; Mathilde ; Robert Courteheuse, fils du duc ; } \\
\text { Guillaume fils du duc ; Jean, évêque d'Avranches. }\end{array}$ \\
\hline
\end{tabular}

Fig. 1 Les souscriptions de Roger II de Montgommery dans les actes ducaux jusqu'en 1066 
Constatons tout d'abord que les bornes chronologiques larges de l'acte sont désormais constituées par l'élévation de Baudouin à l'épiscopat, cérémonie survenue peu après le décès de son prédécesseur, le 20 avril $1066^{54}$, et le départ du duc en Angleterre environ cinq mois plus tard. Entre ces deux dates, on ne retrouve qu'un seul événement où sont rassemblés tous les témoins de la pancarte: la dédicace de l'abbaye de la Trinité de Caen, le 18 juin $1066^{55}$. Il a déjà été noté ailleurs comment les actes de confirmation générale ou les pancartes pour les cathédrales normandes n'ont été délivrés à cette époque que dans des circonstances très particulières ${ }^{56}$, et la cérémonie solennelle de la dédicace de l'abbaye caennaise, qui s'est déroulée dans le sillage de l'assemblée des barons et prélats réunis pour préparer l'expédition d'Angleterre, représente certainement un événement plus que singulier ${ }^{57}$. Les dangers inhérents au projet d'invasion n'avaient pas échappé à l'attention de certains grands du duché ${ }^{58}$, et on se demande si l'évêque d'Avranches, qui comptait parmi les prélats convoqués pour en discuter des préparatifs, avait décidé de mettre en ordre les possessions de sa cathédrale, alors dans un état de grande confusion, et de les faire confirmer par l'autorité ducale en prévision d'un possible échec de l'expédition et des incertitudes qui pourraient en résulter. Nous savons, en effet, que ce fut lors de la dédicace de la Trinité que le duc Guillaume donna la moitié de la terre du Vièvre à la cathédrale d'Avranches ${ }^{59}$, terre que lui avait remise auparavant l'évêque Jean, qui, en l'offrant à son église, voulait la mettre à l'abri des réclamations de ses parents ${ }^{60}$. Entouré non seulement des grands du duché, mais également des fidèles liés à la cathédrale d'Avranches ${ }^{61}$, l'évêque, en assurant la confirmation ducale de la pancarte, semble ainsi avoir profité de la dédicace pour mieux assurer le temporel de son église.

Ce faisant, il imitait son voisin coutançais, le célèbre Geoffroi de Montbray (1048-

\footnotetext{
${ }^{54}$ La Gallia Christiana soutient que l'évêque Guillaume Fleitel, prédécesseur de Baudouin, mourut le 11 février 1066, sans donner de justification à cette information. L'obituaire de la cathédrale d'Évreux marque sa mort au 20 avril. Pour discussion, voir R. Allen, The Norman episcopate, 989-1110, thèse doctorale, University of Glasgow, 2009, dactyl., t. I, p. 223.

${ }_{55}^{5}$ M. Fauroux, Recueil des actes..., $\mathrm{n}^{\circ} 231$.

${ }^{56}$ J. Fontanel, « La réorganisation religieuse... », p. 191.

${ }^{57}$ Sur la dédicace, voir David Bates, William the Conqueror, Londres, 2016, p. 226-227.

${ }^{58}$ The Ecclesiastical History of Orderic Vitalis, éd. Marjorie Chibnall, Oxford, 1969-1980 (Oxford Medieval Texts), t. II, p. 142.

${ }^{59}$ Le Vièvre, Eure, cant. Pont-Audemer, comm. Saint-Philbert-sur-Risle.

${ }^{60}$ M. Fauroux, Recueil des actes..., $\mathrm{n}^{\circ} 229$. On retrouve parmi les témoins de cet acte le duc Guillaume, la comtesse Mathilde, leur fils Robert Courteheuse, l'archevêque Maurille, l'évêque Baudouin, l'évêque Jean et Roger II de Montgommery.

${ }^{61}$ Sur l'identification de certains hommes liés à la cathédrale d'Avranches parmi les témoins de l'acte concernant la terre du Vièvre, voir Richard Allen, «Les actes des évêques d'Avranches de 911 à $1252 »$, dans Revue de l'Avranchin et du pays de Granville, t. 87, 2010, p. 373-380, aux p. 378-380.
} 
1093), qui avait profité, semble-t-il, de la dédicace de la cathédrale de Coutances, le 8 décembre 1056, pour obtenir du duc Guillaume une grande charte confirmant tous les biens de sa cathédrale ${ }^{62}$. Il n'est pas sans intérêt de remarquer aussi que la confirmation ducale de certaines autres pancartes et chartes confirmatives normandes coïncide avec un événement important ${ }^{63}$.

En guise de conclusion, nous ferons simplement quelques remarques. La pancarte de la cathédrale d'Avranches a occupé, depuis sa première publication en 1888, une place centrale dans l'histoire de la réorganisation religieuse de la Normandie occidentale au $\mathrm{XI}^{\mathrm{e}}$ siècle. Sa survie nous permet de connaître plusieurs aspects concrets de l'histoire du diocèse d'Avranches à cette époque, et démontre que l'ouest de la Normandie recèle bien plus de sources qu'on ne l'a dit malgré les catastrophes arrivées depuis la période ducale. Le fait que l'on peut dorénavant situer ce document dans le cadre de l'invasion de l'Angleterre en 1066 ne sert qu'à renforcer son importance. Il nous rappelle tout d'abord que derrière la rédaction d'un tel acte se cachent souvent de nombreux facteurs pratiques, religieux comme politiques. Dans le cas présent, il semble que les développements politiques aient incité l'évêque Jean avant tout à se plonger dans les archives de sa cathédrale, ce qui peut surprendre compte tenu de sa réputation comme réformateur guidé avant tout par des considérations ecclésiastiques. Or, on sait qu'une crise, ou la menace d'une crise, se cache derrière la rédaction de certaines pancartes normandes ${ }^{64}$, depuis que David Bates voit une sorte de symbiose entre les pancartes et chartes confirmatives normandes et la rédaction en Angleterre du Domesday Book, document dont la préparation fut motivée, d'après certains spécialistes, par la menace d'invasion du roi danois Cnut IV (1080-1086) ${ }^{65}$.

Le recours à la rédaction d'un acte écrit nous conduit également à la question de l'autorité du document produit et de son efficacité, ainsi que des éléments qui les

\footnotetext{
62 M. Fauroux, Recueil des actes..., $\mathrm{n}^{\mathrm{o}}$ 214. Pour la suggestion que l'acte, dont les bornes chronologiques larges sont 1056-1066, fut donné à l'occasion de cette cérémonie, voir J. Fontanel, « La réorganisation religieuse... », p. 190.

${ }^{63}$ D. Bates, « Les chartes de confirmation... », p. 104.

${ }^{64}$ Ibid., p. 105.

${ }^{65}$ David Bates, «The Conqueror's charters », dans England in the eleventh century : proceedings of the 1990 Harlaxton Symposium, éd. Carola Hicks, Stamford, 1992 (Paul Watkins medieval studies, 12 ; Harlaxton medieval studies, 2), p. 1-15, aux p. 4-5.
} 
fondent, dont la qualité des personnes qui le présentent et celle des témoins qui le souscrivent ${ }^{66}$. En ce qui concerne le genre de l'acte, il s'inscrit dans une tradition diplomatique déjà bien connue, c'est-à-dire les chartes-pancartes des ducs normands ${ }^{67}$. Mais si, à cet égard, la pancarte avranchaise ne semble, de prime abord, rien offrir d'extraordinaire, la description qu'en donne le vidimus de 1377 est tout à fait particulière, puisque l'exposé de cet acte, tant dans la version retranscrite par le chanoine Guérin que dans la copie du $\mathrm{XVI}^{\mathrm{e}}$ siècle ici mise au jour, évoque un « cirographum seu cartam partitam ${ }^{68}$. L'histoire du développement de la chartepartie, appelée chirographe, qui se développe particulièrement dans le dernier tiers $\mathrm{du}$ $\mathrm{XI}^{\mathrm{e}}$ siècle, a depuis longtemps attiré l'attention des spécialistes ${ }^{69}$. Particulièrement adaptée aux accords, contrats et règlements de conflits, la pratique chirographaire cherche tout d'abord « une stabilisation de l'écriture diplomatique », et la forme et le texte de l'acte, établi en au moins deux exemplaires sur une même feuille de parchemin et coupé selon une légende (tel le mot cyrographum), visent à ce que le

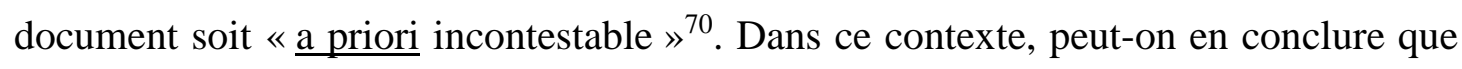
l'évêque Jean, face aux incertitudes de l'invasion de l'Angleterre et au désordre qui régnait jusque-là dans le temporel de sa cathédrale, cherchait à sauvegarder le souvenir de celui-ci non seulement par une pancarte placée sous l'autorité ducale, mais aussi par l'établissement du texte en deux exemplaires ? Compte tenu de l'absence, pour autant que l'on puisse en juger, de tout autre pancarte contemporaine en forme de charte-partie, cela semble peu probable ${ }^{71}$. Et de toute manière, même si l'on croit que l'évêque Jean a cherché à instrumenter un type d'acte totalement

\footnotetext{
${ }^{66}$ Sur cette question, voir L'autorité de l'écrit au Moyen Âge (Orient - Occident) : XXXIX ${ }^{\mathrm{e}}$ congrès de la SHMESP (Le Caire, 30 avril - 5 mai 2008), Paris, 2009 (Histoire ancienne et médiévale, 102).

${ }^{67}$ Michel Parisse, "Écriture et réécriture des chartes : les pancartes aux $\mathrm{XI}^{\mathrm{e}}$ et $\mathrm{XII}^{\mathrm{e}}$ siècles », dans Bibliothèque de l'École des chartes, t. 155, 1997, p. 247-265, aux p. 250-252 ; D. Bates, « Les chartes de confirmation... », p. 95-109.

68 «Universis presentes litteras inspecturis, officialis Abrincen(sis), salutem in Domino. Notum facimus quod anno Domini millesimo trecen(tissim)o sep(t)uage(sim)o septimo, decima die mensis jullii, nos vidimus et diligenter inspeximus quendam cirographum seu cartam partitam spectan(tem) et pertinen(tem) ecclesie Abrincen(si) et preben(dis) et personis ejusdem ecclesie, cujus quidem cirographi seu carte tenor sequitur in hec verba... », Bibl. mun. Avranches, fonds Pigeon, ms. 45 (26bis), p. 448.

${ }^{69}$ Michel Parrise, «Remarques sur les chirographes et les chartes-parties antérieures à 1120 et conservées en France », dans Archiv für Diplomatik, t. 32, 1986, p. 546-567.

${ }^{70}$ Laurent Morelle, «Instrumentation et travail de l'acte : quelques réflexions sur l'écrit diplomatique en milieu monastique au XI ${ }^{e}$ siècle », Médiévales, 56, 2009, p. 41-74, à la p. 65.

${ }^{71}$ Il n'en existe aucun exemple parmi les 5032 chartes originales antérieures à 1121 conservées en France (http://www.cn-telma.fr/originaux/index/). Je tiens également à remercier Chantal Senséby, Dominique Stutzmann et Soline Kumaoka de leurs conseils précieux sur le soi-disant pancarte en forme de charte-partie d'Avranches, qui semble exceptionnelle à leur connaissance.
} 
novateur, on se demande auquel le second exemplaire aurait été confié (aux archives ducales, dont on ne possède pas la moindre trace au $\mathrm{XI}^{\mathrm{e}}$ siècle ?). On est ainsi conduit à admettre qu'il s'agit plus probablement d'une erreur d'interprétation, néanmoins fascinante, de la part du scribe du vidimus.

Quoi qu'il en soit, il n'est pas surprenant que le duc Guillaume occupe dans l'acte une place centrale. Non seulement la pancarte valorise-t-elle sa générosité et celle de ses prédécesseurs, en particulier celle de son père, Robert le Magnifique, mais la souscription ducale sert à marquer sa participation tangible à la restauration et à la remise en ordre du temporel de la cathédrale. La pancarte s'inscrit ainsi dans la politique constante des princes normands, marquée par une ferme volonté de réorganisation religieuse, mais à condition de rester maîtres de la situation. Il est également à noter que la pancarte est l'un des rares actes souscrits par plusieurs membres de la famille ducale (Guillaume, Mathilde et plusieurs fils), ainsi que par l'épiscopat normand : il n'y a en effet que deux autres exemples, dont l'un fut donné lors de la dédicace de la Trinité de $\mathrm{Caen}^{72}$, ce qui non seulement renforce l'hypothèse que la pancarte fut confirmée lors d'un grand événement tel qu'une dédicace abbatiale, voire la dédicace de la Trinité, mais encore que l'évêque Jean, face aux incertitudes liées au projet d'invasion, voulait assurer aussi bien que possible sa validité. Conscients de ces incertitudes, d'autres ecclésiastiques ont également cherché à la veille de l'invasion la confirmation de leurs biens par Guillaume et son héritier officiel, Robert Courteheuse ${ }^{73}$, qui, avec l'appui de sa mère et certains seigneurs normands, dont Roger de Montgommery, devait gouverner la Normandie en l'absence du duc ${ }^{74}$. La pancarte avranchaise témoigne ainsi non seulement d'une remise en ordre importante des biens d'une cathédrale normande, mais aussi des préparatifs considérables entrepris dans toute le duché, ainsi que dans ses environs, en vue de la conquête de l'Angleterre.

Or, si la pancarte constitue aujourd'hui le fondement documentaire de la mémoire historique et religieuse du diocèse, elle ne semble jamais avoir occupé une place

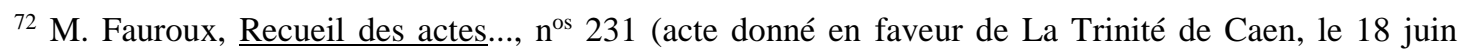
1066) et 232 (acte donné en faveur du Mont Saint-Michel, entre le 27 mai et le 16 juillet 1066).

${ }^{73}$ Ibid., $n^{\circ} 228$ (acte confirmant à l'abbé de Marmoutier Barthélemy toutes les donations que le duc Guillaume avait accordées à cette abbaye). On retrouve Jean, évêque d'Avranches, et Roger II de Montgommery parmi les témoins de cet acte.

${ }^{74}$ Sur les préparatifs politiques de l'invasion, voir D. Bates, William the Conqueror..., p. 219-221.
} 
significative dans la mémoire archivistique ancienne de la communauté avranchaise. Notons tout d'abord qu'à la différence de nombreuses grandes chartes confirmatives contemporaines, dont celle qui fut adressée à la cathédrale de Coutances, le texte de la pancarte n'a jamais été transcrit dans un cartulaire ${ }^{75}$. L'acte ne paraît en outre jamais avoir servi comme pièce à conviction lors d'un procès et, parce que l'auteur juridique en est l'évêque, et non le duc, son texte n'a jamais été inséré dans un vidimus royal ${ }^{76}$. Sur les raisons pour lesquelles la pancarte fit en 1377 l'objet d'un vidimus d'officialité nous en sommes malheureusement réduits à des conjectures, mais il est possible qu'elle ait été en mauvais état, comme ce fut le cas de la charte coutançaise ${ }^{77}$. Nous ignorons le moment exact où la pancarte fut perdue, mais elle fut probablement parmi les «chartres, lettres et enseignements » brulées en 1562, lors du sac de la cathédrale par les forces huguenotes ${ }^{78}$. Quoi qu'il en soit, au vu des pertes et destructions infligées par les protestants, on ne saurait être surpris par le fait que les archives diocésaines n'ont guère suscité l'intérêt des érudits qui firent plus tard le tour de la région pour se plonger dans les riches archives que conservaient beaucoup d'autres institutions ecclésiastiques locales (Le Mont Saint-Michel, Savigny, Lessay, Saint-Sauveur-le-Vicomte...). Face à l'état fragmentaire de la documentation, ceux qui s'y sont intéressés ont parfois introduit des erreurs dans l'histoire de l'Église avranchaise, dont le chanoine Guérin et l'abbé Pigeon qui ont tous les deux attribué la rédaction de la pancarte à l'évêque Jean de Hautfumé $(1331-1358)^{79}$. Quant à nous, nous ne pouvons malheureusement que déplorer les pertes archivistiques de la cathédrale d'Avranches, intervenues depuis des siècles. Nous espérons néanmoins avoir ouvert quelques perspectives nouvelles sur l'un de ses trésors documentaires et fait progresser quelque peu la compréhension tant de la réorganisation ecclésiastique du diocèse $\mathrm{au} \mathrm{XI}^{\mathrm{e}}$ siècle que de l'histoire plus large de la Normandie ducale.

\footnotetext{
${ }^{75}$ L'abbé Pigeon, qui a fait son édition d'après la copie faite par le chanoine Guérin du vidimus de 1377, a inclus sa transcription de la pancarte dans sa « reconstitution » du cartulaire perdu dit Livre blanc, sans donner de justification (É.-A. Pigeon, Le diocèse d'Avranches..., t. II, p. 666).

${ }^{76}$ À l'inverse, l'acte donné par le duc concernant la terre du Vièvre fit l'objet d'un vidimus royal en juin 1338 et fut copié dans le cartulaire dit Livre blanc (M. Fauroux, Recueil des actes..., $\mathrm{n}^{\circ}$ 229).

${ }^{77}$ Le vidimus ne donne aucun renseignement sur les caractères externes de l'acte vidimé (voir ci-dessus n. 000). Sur la charte de Coutances, voir J. Fontanel, « La réorganisation religieuse... », p. 191.

${ }^{78}$ F. Gueroult, "Procès-verbal du pillage de la cathédrale et de l'évêché d'Avranches par les Protestants, en 1562 », dans Revue de l'Avranchin, t. 9, 1898, p. 147-171, à la p. 167.

79 Bibl. mun. Avranches, fonds Pigeon, ms. 45 (26bis), p. 451; É.-A. Pigeon, Le diocèse d'Avranches..., t. II, p. 333, 666 .
} 


\section{Annexe}

[1066, 18 juin. — Caen].

Guillaume le Bâtard souscrit la pancarte en forme de charte récapitulative par laquelle Jean [d'Ivry], évêque d'Avranches, énumère les possessions de sa cathédrale. Ayant pris connaissance des chartes de l'église et y ayant trouvé de grandes confusions dans les possessions de celle-ci (certaines chartes faisaient référence à des biens qui n'appartenaient pas à l'église, d'autres ignoraient dans leur texte des biens dont elle avait la propriété ; il résultait en effet de ces chartes qu'il y avait un échange fait à la $\underline{\text { suggestion et sur l'ordre du très glorieux prince Guillaume), l'évêque a fait au mieux }}$ les corrections qui s'imposaient, en collationnant les chartes qui, mélangées avec $\underline{\text { d'autres, étaient par trop fautives. C'est d'abord la terre des Cresnays }}{ }^{80}$ avec ses dépendances, ainsi que l'église que [le comte] y possédait anciennement sans $\underline{\text { contestation, la terre de Pontaubault }}^{81}$, celle aussi de [Saint-Jean-de-]la-Haize ${ }^{82}$, avec $\underline{\text { Gée }}^{83}{ }_{2}$ [Asinguer] et les autres dépendances, le bourg de Ponts ${ }^{84}$ avec Mallouée $^{85}$ et ses autres dépendances, c'est-à-dire les moulins, les prés et l'eau jusqu'à la Roche$\underline{\text { Rompue }}^{86}$ et les terres autour et en-dessous de la ville, et la terre de Celland ${ }^{87}$ avec ses forêts, prés, eaux et terres cultivées et non cultivées, la terre que l'on appelle Champeaux, et la terre de Juilley ${ }^{88}$ avec ses églises et toutes ses dépendances, c'est-à$\underline{\text { dire Poilley }}^{89}$, Précey $^{90}$ et la terre (...), toutes terres que le comte Richard [II] avait possédées de tout temps et qu'il a octroyées à l'église d'Avranches. L'évêque énumère ensuite les terres, églises, dîmes et moulins que le prince Robert, fils du comte Richard, a concédés à la cathédrale Saint-André, à la demande d'Hugues, évêque d'Avranches : l'église de Saint-Gervais dans la banlieue d'Avranches, avec la dîme de la paroisse et avec les biens que les clercs de l'église possédaient alors, et

\footnotetext{
${ }^{80}$ Manche, cant. Isigny-le-Buat.

${ }^{81}$ Manche, cant. Pontorson.

${ }^{82}$ Manche, cant. Avranches.

${ }^{83}$ Manche, cant. Avranches, comm. Saint-Jean-de-la-Haize.

${ }^{84}$ Manche, cant. Avranches.

${ }^{85}$ La carte de Cassini montre cet ancien faubourg de Ponts à l'endroit où se trouve actuellement la ZA du Motté.

${ }^{86}$ Soit La Roche, Manche, cant. Pontorson, comm. Le Val-Saint-Père, soit La Roche-Torin, Manche, cant. Pontorson, comm. Courtils.

${ }^{87}$ Manche, cant. Isigny-le-Buat, comm. Le Grand- et Le Petit-Celland.

${ }^{88}$ Manche, cant. Pontorson.

${ }^{89}$ Manche, cant. Pontorson.

${ }^{90}$ Manche, cant. Pontorson.
} 
$\underline{\text { trois églises avec leurs dîmes, l'une dans le village des Gens }}{ }^{91}$ avec une charruée de $^{\text {ave }}$ $\underline{\text { terre, la deuxième dans celui de Céaux }}^{92}$ avec une charruée de terre, et la troisième dans celui de Vessey ${ }^{93}$ avec une charruée de terre; puis quatre autres églises avec leurs dîmes, à savoir l'église de la Croix[-Avranchin] ${ }^{94}$, l'église de Villiers[-le-Pré] ${ }^{95}$, l'église de Saint-Senier[-de-Beuvron] $^{96}$, dont la paroisse comporte également un $\underline{\text { moulin sur le Beuvron, et l'église de la paroisse de Vains }}{ }^{97}$ avec un manse de terre et une terre de deux hommes francs. Ce même prince lui a concédé aussi la dîme de tout $\underline{\text { le tonlieu de l'Avranchin et la dîme de deux foires annuelles, dont l'une se tient à la }}$ Croix[-Avranchin], depuis lors transférée à Saint-James ${ }^{98}$, et l'autre à Avranches, le jour de la Saint-André. Il a également concédé la terre du clerc Gautier, fils de $\underline{\text { Morin, qui s'appelle Noirpalu }}^{99}{ }^{\text {, avec l'église et les moulins, et une partie du village }}$ de Champeaux $^{100}$, avec la moitié de l'église. Il a donné également la Champagne ${ }^{101}$ $\underline{\text { avec deux moulins et avec une partie de la forêt, et le village de Plomb }}{ }^{102}$ et celui de $\underline{\text { Braffais }}^{103}$ avec les églises et les moulins, et un mesnil avec un moulin aux Gens. Le comte Robert a également donné la terre de Celland, avec ses forêts et ses cours d'eau, ses églises et ses moulins, ses terres cultivées et non cultivées. Il a donné de même le tonlieu perçu dans tout l'évêché ; il a donné de même Chassilly ${ }^{104}$ et Saint$\underline{\text { Pois }}^{105}$, c'est-à-dire la terre de Guillaume Silvain ; mais le fils [du comte], Guillaume, a rendu cette terre au comte de Mortain, et il a donné en échange à la cathédrale la terre du clerc Giraud, frère de Godeflel, c'est-à-dire le village de Saint-Senier[-sousAvranches $^{106}{ }^{10}$ la terre d'Appilly ${ }^{107}$ et d'Orceil $^{108}{ }^{\text {avec les églises et les dîmes de son }}$ patrimoine et la terre de Ronthon ${ }^{109}$, c'est-à-dire Saint-Pierre[-Langers] ${ }^{110}$,

\footnotetext{
${ }^{91}$ Manche, cant. Pontorson, comm. Saint-Quentin-sur-le-Homme.

${ }^{92}$ Manche, cant. Pontorson.

${ }^{93}$ Manche, cant. Pontorson.

${ }^{94}$ Manche, cant. Saint-Hilaire-du-Harcouët.

${ }^{95}$ Manche, cant. Saint-Hilaire-du-Harcouët.

${ }^{96}$ Manche, cant. Saint-Hilaire-du-Harcouët.

${ }^{97}$ Manche, cant. Avranches.

${ }^{98}$ Manche, cant. Saint-Hilaire-du-Harcouët.

${ }^{99}$ Manche, cant. Villedieu-les-Poêles, comm. Le Tanu.

${ }^{100}$ Manche, cant. Avranches.

${ }^{101}$ Manche, cant. Avranches, comm. Plomb.

102 Manche, cant. Avranches.

${ }^{103}$ Manche, cant. Isigny-le-Buat.

${ }^{104}$ Manche, cant. Saint-Hilaire-du-Harcouët, comm. Saint-Senier-de-Beuvron.

105 Manche, cant. Villedieu-les-Poêles.

${ }^{106}$ Manche, cant. Isigny-le-Buat.

${ }^{107}$ Manche, cant. Isigny-le-Buat, comm. Saint-Senier-sous-Avranches.

${ }^{108}$ Manche, cant. Isigny-le-Buat, comm. La Gohannière.

${ }^{109}$ Aujourd'hui Dragey-Ronthon, Manche, cant. Avranches.
} 
$\underline{\text { Chantore }}^{111}$ et [Frigabulgam]. Il a également concédé, au lieu du Mesnil-Gilbert ${ }^{112}{ }_{2}$ $\underline{\text { Saint-Ovin }}^{113}$ avec l'église et les moulins, les terres cultivées et non cultivées et toutes ses dépendances. L'église d'Avranches possède également, d'ancienneté, deux $\underline{\text { domaines dans le Cotentin, l'un qui s'appelle Coutainville }}^{114}$ et l'autre Vallière $^{115}$, et $\underline{\text { toutes les églises de la ville et de ses faubourgs. Le comte Guillaume a également }}$ donné, à la demande dudit évêque d'Avranches Hugues, toute la terre que tenait Garnier, frère du portier Thierry, dans l'Avranchin. Baudouin, fils du comte Gilbert [de Brionne], avec la permission du prince Guillaume, a également donné à ladite église, sur la demande de Jean, évêque d'Avranches, plusieurs parcelles de terre qu'il possédait sur la rive du Saultbesnon, lesquelles parcelles ont été incluses par l'évêque $\underline{\text { dans le parc qu'il faisait construire à grands frais }}{ }^{116}$. Le prince Guillaume a également donné à l'église d'Avranches, à la demande de l'évêque Jean, la dîme du tonlieu de Mayenne et celle du passage et de la taxe du grain.

A. Original perdu.

B. Copie du 10 juillet 1377 dans un vidimus perdu de l'officialité d'Avranches (d'après $\underline{\mathrm{A}}$ ). $-\underline{\mathrm{C}}$. Copie perdue du 10 avril 1540 faite par Pierre Gaudin et Lancelot Philippes, tabellions royaux (d'après $\underline{B}$ ). - - D. Copie du XVI ${ }^{\mathrm{e}}$ siècle, Bibl. mun. Avranches, fonds Pigeon, ms. 45 (26bis), p. 448-450 (d'après $\underline{\text { C). }}$ - E. Copie du XVII ${ }^{\mathrm{e}}$ siècle, Bibl. mun. Avranches, fonds Pigeon, ms. 45 (26bis), sur des feuillets intercalés entre p. 449 et 450 (traduction française de $\underline{\mathrm{C}}$ ). $-\underline{\mathrm{F}}$. Copie du XVII siècle par Charles Guérin, Bibl. mun. Avranches, fonds Pigeon, ms. 45 (26bis), p. 451-453 (d'après une copie de $\underline{B})^{117}$.

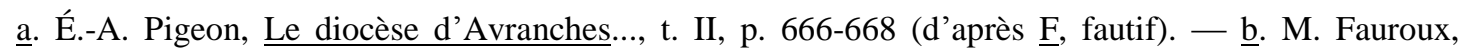
Recueil des actes..., p. 24 n. 24, p. 25-26, n. 29, p. 27 nn. 33 et 37 (extraits, d'après $\underline{\text { a). }}$ - $\underline{\text { c. }}$ R. Allen, «Un évêque et sa ville... », $n^{\circ}$ II, p. 38-44 (d'après $\underline{\text { F). }}$

La tradition textuelle de cet acte est très défectueuse : en particulier, la fin de la phrase qui commence par «imprimis» («terram que intime possiderat que Richardus ... tradidit») est manifestement fautive; Pigeon l'avait reconnu en dotant chacun des deux «que»d'un sic, mais sans proposer de

\footnotetext{
${ }^{110}$ Manche, cant. Avranches.

${ }^{111}$ Manche, cant. Avranches, comm. Bacilly.

${ }^{112}$ Manche, cant. Isigny-le-Buat.

113 Manche, cant. Pontorson.

${ }^{114}$ Aujourd'hui Agon-Coutainville, Manche, cant. Saint-Malo-de-la-Lande

${ }^{115}$ Manche, cant. Saint-Malo-de-la-Lande, comm. Blainville-sur-Mer.

${ }^{116}$ Le Parc, Manche, cant. Isigny-le-Buat, comm. Braffais.

117 «Ex copia fidelissima per quendam [...] in arch. mihi communicata », Bibl. mun. Avranches, fonds Pigeon, ms. 45 (26bis), p. 451 (en marge).
} 
solution, et Fauroux (p. 24, n. 24) a repris le texte de Pigeon sans même conserver les $\underline{\text { sic. }}$. Et tous deux ont traité la phrase « imprimis » comme un ensemble analogue à ceux d' «inscripsimus deinde » et des divers «dedit etiam» et «concessit etiam» qui suivent, bien qu'elle soit dépourvue de verbe principal ; nous la voyons plutôt comme la liste des biens impliqués dans le désordre consécutif à la «mutatio » effectuée à la suggestion de Guillaume. La correction du premier «que » en «quam» n'étant pas suffisante pour reconstruire la phrase, nous proposons de conclure à l'existence d'une lacune dans l'identification de «terram», de présumer comme antécédent de «que » un «omnia » récapitulatif, exprimé ou sous-entendu, et de faire de «Richardus » le sujet des deux verbes, tout en étant conscient que c'est prendre parti sur le contenu de son patrimoine originel.

\section{Texte établi d'après $\underline{\mathrm{D}}$}

Q(uonia)m multa torpore et negligentia pereunt que, si ordine suo subsisterent ut deceret, vigerent, ego Joannes quamvis indignus sancte Abrincen(sis) ecclesie episcopus, cartas ecclesie nostre respiciens ${ }^{(a)}$ magnamque terrarum confusionem in eis inveniens (quamplures enim terrarum possessiones quas ecclesia non habebat carte intra se continebant, quasdam vero quas habebat inscriptione sua ecclesiam habere denegabant ; erat enim ex eis facta mutatio Guill(elm)i gloriosissimi principis hortatu et jussu), que pluribus cartulis confuse titubabant nimium colligens summa veritate correximus ut decebat: imprimis terram Cresney cum suis appendiciis, cum ecclesia quam antiquitus absque calumnia possederat, et terram Pontis Alboldi, terram quoque Haisa cum Gagiaco et $\underline{\text { Asinguer }}^{(\mathrm{b})}$ ceterisque appendiciis, et burgum Pontis cum Maloiaco ceterisque appendiciis, scilicet molendinis et pratis et aqua usque ad Rupem Necatam, et terras in circuitu civitatis et infra, et terram Serlant cum silvis, pratis et aquis, terris cultis et incultis, et terram que Campellis vocatur, et terram Ilgeon cum ecclesiis et omnibus ${ }^{(\mathrm{c})}$ appendiciis suis, scilicet Polei ${ }^{(\mathrm{d})}$, Pressei et terram [...] que intime possederat ${ }^{(\mathrm{e})}$ Richardus comes <et> ecclesiae Abrincen(si) tradidit. Inscripsimus deinde terras, ecclesias, decimas, molendina que Robertus nobilissimus princeps, filius Richardi comitis, qui $^{(\mathrm{f})}$ zelo divini amoris succensus Hierosolimis sepulturam dominicam visitavit, beato Andree prece et hortatu Hugonis venerabilis antistitis concessit, id <est> in suburbiis civitatis ecclesiam Sancti Gervasii, cum decima parrochie et cum possessionibus quas clerici ecclesie tunc possidebant, tresque ecclesias cum decimis : unam in villa que dicitur Esgen cum terra unius carruce, alteram in villa Celsi ${ }^{(\mathrm{g})}$ cum terra unius carruce, tertiam in villa que dicitur Vecei cum terra unius carruce; quatuor quoque alias ecclesias cum decimis, scilicet ecclesiam Crucis et ecclesiam ville Vileris et ecclesiam Sancti Senerii in cujus parochia super 
ripam fluminis Bevronis, molendinum pariter tribuit et ecclesiam ville que dicitur $\underline{\mathrm{Veyn}}^{(\mathrm{h})}$ cum uno manso terre et terram duorum francorum. Concessit etiam decimam totius telonei $\mathrm{Ab}$ (rincensis) pagi, et decimam de duabus nundinis anualibus, quarum una $^{(i)}$ hab(ebatur) tunc in villa Crucis, modo mutata apud Sanctum Jacobum, altera vero in festivitate sancti Andree Abrincis. Concessit etiam sancti Andree terram Gualterii clerici Morini filii, que vocatur Nigrapalus cum ecclesia et molendinis, et partem ville que dicitur Campellis cum medietate ecclesie. Dedit etiam Campaniam cum duobus molendinis et cum una parte silve et villam que vocatur Plom, et alteram que vocatur $\underline{\text { Braffays }}^{(\mathrm{j})}$ cum ecclesiis et molendinis et unum mosnille ${ }^{(\mathrm{k})}$ cum uno molendino in villa que dicitur Esgen. Dedit etiam Robertus comes terram que vocatur $\underline{\text { Serlant, }}$ cum silvis et aquis, ecclesiis et molendinis ${ }^{(1)}$, cultis et incultis. De omnicumque episcopatu teloneum similiter dedit. Castiniacum vero et Sanctum

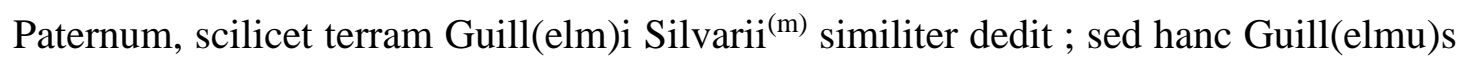
ejus filius comiti Morethonii ${ }^{(n)}$ reddens, pro ea terram Giraldi clerici fratris Godefleli scilicet Sancti Senerii villam, terram ${ }^{(0)}$ Appiliacum et Orsolum, cum ecclesiis et decimis patrimonii sui quas tenebat et terram Rontonis scilicet Sanctum Petru(m) et Cantorias et Frigabulgam in scambio tradidit. Pro Maisnilo ${ }^{(\mathrm{p})}$ Gilberti Sanctu(m) Audoinum cum ecclesia et molendinis, terris cultis et incultis et omnibus appendiciis concessit. Possidet etiam antiquitus ecclesia Abrincen(sis) in Constantiensi pago duas villas, una que vocatur Constantis Villa ${ }^{(\mathrm{q})}$, altera Valeria, et omnes ecclesias civitatis et suburbii. Dedit etiam comes Guill(elmu)s, precatu Hugonis prefati episcopi Abrincen(si) ecclesie, omnem terram quam tenebat Garnerius frater Theodorici hostiarii in Abrincen(si) pago. Dedit etiam Balduinus filius Guilberti comitis, concedente Guill(elm)o principe, prefate ecclesie, particulas terre quas in circuitu rivuli qui Limon vocatur possidebat, prece et hortatu Joannis episcopi, quas particulas idem episcopus infra Parcum quem cum pecunia construxerat inclusit. Dedit etiam Guill(elmu)s princeps decimam telonei ${ }^{(\mathrm{r})}$ Meduane et transitus et minagii, prece Joannis episcopi ${ }^{(\mathrm{s})}$, ecclesie Abrincen(si).

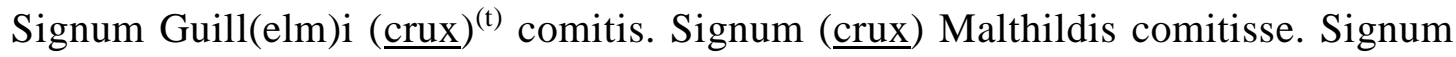
( $\underline{\text { rux }})$ Roberti comitis. Signum ( $\underline{\text { crux }})$ Richardi fratris <ejus>. Signum (crux) Maurilii archiepiscopi. Signum ( $\underline{\text { rrux }}$ ) Balduini <Ebroicensis episcopi>. Signum (crux) Joannis ${ }^{(\mathrm{u})}$ Abrincen(sis) episcopi. Signum (ㄷux $)$ Rogerii Montis $\underline{\text { Grivou }}^{(\mathrm{v})}$. 
(a) magnamque barré D. - (b) Ce mot est difficile à lire en F; Pigeon a suggéré Asingucriis - (c) omnibus inséré en interligne D. - (d) Pollei F. - (e) DF ajoutent que, suivi de Richardus barré F. -

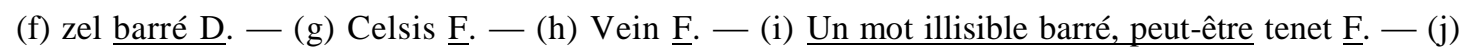
Braffais F. - (k) Sic DF, comprendre mesnil - (l) terris ajouté F. - (m) Silvani F. - (n) Moretonii F. - (o) terram omis et Api. barré F. - (p) Mesnillo F. - (q) Constantis-villa F. — (r) Premier jambage de la lettre $\mathrm{m}$ barré, F. - (s) episcopi inséré en interligne D. - ( $\mathrm{t}$ ) Le copiste de D $\underline{\text { signale les croix de souscription avec les mots une croix. - (u) Signum (crux) Joannis inséré en }}$ interligne D. - (v) Liste des souscriptions omise F. 